Musées, Patrimoine et Culture scientifiques et techniques

\title{
De la dissimulation à la révélation : les visiteurs
} face à la technologie RFID

Nathalie Candito et Fabrice Forest

\section{OpenEdition Journals}

Édition électronique

URL : http://journals.openedition.org/ocim/720

DOI : $10.4000 /$ ocim.720

ISSN : 2108-646X

Éditeur

OCIM

Édition imprimée

Date de publication : 1 septembre 2007

Pagination : 18-24

ISSN : 0994-1908

Référence électronique

Nathalie Candito et Fabrice Forest, « De la dissimulation à la révélation : les visiteurs face à la

technologie RFID », La Lettre de I'OCIM [En ligne], 113 | 2007, mis en ligne le 09 février 2011, consulté le 19 avril 2019. URL : http://journals.openedition.org/ocim/720 ; DOI : 10.4000/ocim.720 
qualitative inspirée de la méthode CAUTIC ${ }^{(2)}$ qui permet d'observer comment et pourquoi les utilisateurs adoptent ou non un dispositif technologique nouveau. Pour l'utilisateur, toute proposition technologique arrive dans un contexte constitué :

- des techniques existantes de l'utilisateur, c'est-àdire les savoirs, les savoir-faire d'utilisation et les manières de faire ;

- de pratiques coutumières de l'utilisateur, c'est-àdire les habitudes, les activités et les besoins liés au mode de vie ;

- de l'identité de l'utilisateur, c'est-à-dire le rôle (privé ou professionnel) et le système de valeurs personnelles ;

- de son environnement social, c'est-à-dire le milieu social et culturel et les formes de sociabilités associées. L'approche CAUTIC permet d'explorer et d'analyser les représentations des utilisateurs confrontés à un dispositif innovant pour déterminer le sens de l'usage. Pour «faire sens » auprès des utilisateurs, une proposition novatrice doit provoquer des significations d'usage positives dans leurs représentations et leurs manières de vivre cette innovation.

Une enquête exploratoire par interviews (3) a été menée auprès des visiteurs munis de la carte RFID (soit « usagers » à leur insu), à la sortie de l'exposition Ni vu ni connu, à l'endroit de la borne de sortie destinée à la remise de la carte. La trame des entretiens semi-directifs permet d'explorer les quatre niveaux de significations d'usage du dispositif (techniques, pratiques, identité et environnement social de l'utilisateur) et intègre nos hypothèses relatives à la spécificité du dispositif testé :

- réactions spontanées sur l'expérience de visite,

- usages de la carte (ergonomie...),

- compréhension du concept (traçabilité...),

- droit et vie privée (partenariat CNIL),

- apports, limites, prolongement de l'expérience de visite.

\section{Préambule et constats}

\section{Les biais conceptuels}

Au cours des entretiens, un problème de compréhension du « dispositif tag RFID » dans la visite de l'exposition est apparu, créant une ambiguïté dans la perception du concept. Les visiteurs ont du mal à dissocier le rôle joué par les tags RFID du rôle joué par la photographie et confondent le dispositif testé avec un dispositif de sécurité de type vidéo-surveillance. Dans l'esprit des visiteurs, les deux technologies (traçage RFID + image) ne font plus qu'un.
La compréhension du concept de médiation basé sur les RFID s'en trouve perturbée. Comme par exemple, cette visiteuse pensant être photographiée puis interrogée parce qu'elle a enfreint le règlement concernant la prise de photographies : "ça me fait rire. Parce que je prends toujours des photos dans les expos, et là on s'est vu là en fait (...) c'est bien, et même pour traquer les photographes c'est pas mal (...) Voilà, c'est bien mais en même temps qu'est-ce que vous cherchez par rapport aux gens qui prennent des photos? (...) Moi j'ai cru que j'allais avoir une amende pour avoir pris des photos » (femme avec enfant, 35-44 ans, artiste).

Cette association d'idées liée aux technologies employées est à prendre en compte dans l'analyse des représentations sécuritaires que nous avons observées dans les entretiens et qui ont pu s'en trouver renforcées.

\section{Ergonomie et format de la carte}

Bien que l'entretien ne se focalisait pas sur les aspects ergonomiques liés aux conditions de réalisation de l'expérience, les utilisateurs ont spontanément abordé des problèmes d'utilisation liés à l'ergonomie du dispositif. Le format de la carte distribuée a posé des problèmes d'utilisation aux visiteurs. Son encombrement doit pouvoir se justifier : elle doit être ni trop petite (sinon l'utilisateur oublie de s'en servir ou redoute la perte), ni trop grande (car elle constitue une gêne au cours de la visite) : « ... comme c'est un objet qu'on nous remet à l'accueil on a l'impression qu'elle va nous servir davantage à la compréhension de l'exposition et surtout en quantité. Et donc on reste un petit peu sur sa faim, en se demandant pourquoi on a eu ça dans les mains tout le temps » (femme, 25-34 ans, cadre supérieur).

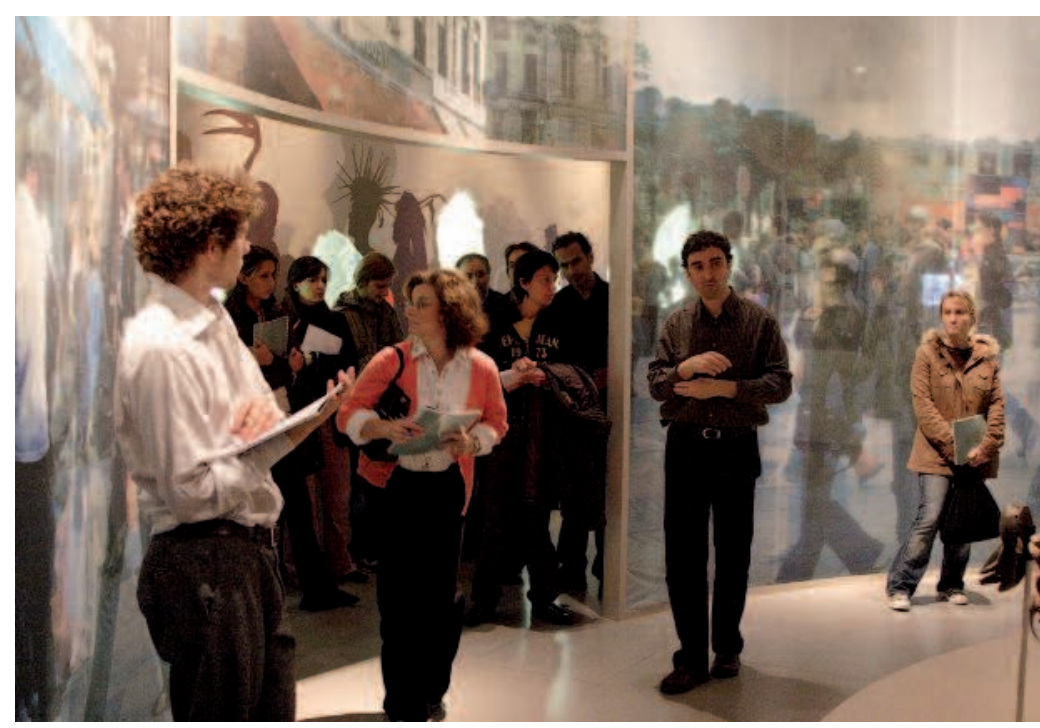

Le public pénètre dans la partie de l'exposition traitant des masques. ๑) Muséum-Département du Rhône/Patrick Ageneau 
Au-delà du format, le principe même de la carte pose un problème d'ergonomie cognitive dans le sens où elle perturbe la concentration ou la liberté d'esprit du visiteur pendant la visite en l'obligeant à penser à se servir de cette carte, ou au moins, à ne pas la perdre : " on stresse si on la perd [...] Au début on se dit c'est marrant je l'ai pas encore utilisé, y'aura encore d'autres choses après, c'est pas mal, ouais c'est bien 》 (2 femmes, 25-34 ans/18-24 ans, banque, étudiante droit).

En conséquence, le dispositif RFID remis au visiteur (carte, ticket ou autre) ne doit pas occasionner une gêne supplémentaire à la visite du musée. S'il s'agit d'un dispositif spécial ou encombrant, la gêne doit se justifier par l'usage au cours de la visite (fréquence et valeur ajoutée à l'expérience de visite).

Une proportion importante de visiteurs dans notre échantillon n’a pas accompli le parcours idéal (bornes manquées, en panne, occupées, difficultés d'utilisation, désintérêt, passage à toutes les bornes et réponse à toutes les questions). Pour certains utilisateurs, le problème était directement lié à l'utilisabilité des bornes car même s'ils ont eu accès à ces dernières, ils n'ont pas été en mesure de les utiliser pour des raisons ergonomiques ou cognitives : «je crois qu'on a rien compris au truc » (couple, femme, 5564 ans, profession libérale ; homme, +65 ans, retraité).

Par conséquent, il est préférable de ne pas faire reposer l'interactivité ou l'immersion de l'utilisateur sur la seule interaction avec des bornes.

\section{Cadre juridique}

Notre questionnement sur la technologie sous-tendait également des aspects liés au cadre juridique dans lequel s'inscrivent les pratiques et usages des dispositifs. L'absence quasi-totale de référence spontanée à un encadrement juridique de l'utilisation des technologies de contrôle est l'un des résultats les plus net de l'enquête.

Le besoin d'exercer un contrôle sur les données personnelles enregistrées par le système est très faible et se décline sous deux formes majeures : une posture renvoyant à la dérision ou indifférence («noyé dans la masse »)ou une posture relevant du fatalisme (inexorable, "on est déjà fiché»).

\section{De l'intégration des technologies dans l'exposition à l'appropriation par les visiteurs}

\section{Méconnaissance de la technologie RFID}

Quand cette technologie est évoquée par l'enquêteur, la majorité des visiteurs a entendu parler des RFID sans être capable de décrire précisément ce

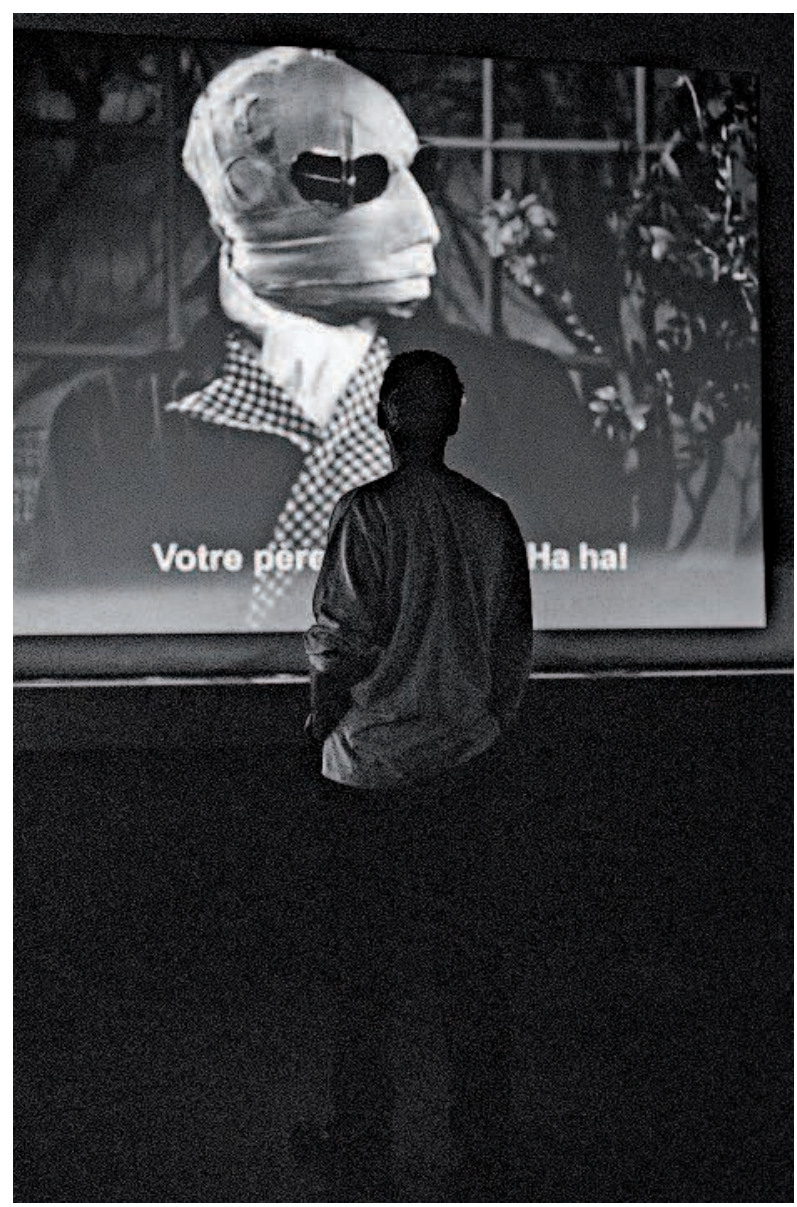

Invisibilité, mythes et fictions : un visiteur regarde un montage d'extraits du long métrage L'homme invisible (1933).

(c) Muséum-Département du Rhône/Bruno Lapray

dont il s'agit. En effet, peu d'interviewés sont déjà informés sur la technologie RFID avant l'interview. Lorsque c'est le cas, les applications évoquées montrent une confusion avec les technologies de géo localisation aux applications sécuritaires, comme la surveillance de condamnés, militaires, ou scientifiques, comme l'observation de la faune. L'imaginaire collectif lié à cette technologie est également très influencé par la littérature et le cinéma de fiction qui constitue un cadre commun de références. Lorsque que l'explication du système RFID est dévoilée au visiteur, apparaissent des références à la biométrie, la biotechnologie, au flicage (fictio : " big brother », " Minority report »), à la vidéosurveillance... des applications largement exploitées par la fiction et qui ont généré ou formaté un imaginaire fortement connoté. Les représentations associées aux RFID ou cartes à puces sont négatives, face à des significations d'usage qui se révéleront relativement positives dans le cadre du musée : "moi un jour j'ai un ami, qu'est un peu un marginal, qui m'a dit « attention pense à moi le jour où on mettra 
une puce sous la peau des gens ». Ça m'a fait penser à ça, un truc qui permet de nous suivre finalement partout où on ira » (femmes, 25-34 ans/18-24 ans ; banque, étudiante droit). La plupart des interviewés perçoivent d'emblée le dispositif RFID dans le musée comme un système de comptage et de traçage à l'image des applications de ces puces dans d'autres domaines : applications sécuritaires, statistiques, marketing...

\section{Relation de méfiance à la technologie}

La compréhension du dispositif technique s'accompagne d'une compréhension spontanée des problèmes éthiques et de vie privée. L'expérience antérieure qu'ont les interviewés des dispositifs de traçabilité intrusifs comme le «spam » (4) les a sensibilisés aux risques associés à ce type de technologie pour leur vie privée. Cette expérience personnelle est l'une des raisons pour lesquelles certains visiteurs hésitent à laisser leur adresse e mail au musée : " c'est assez sympa mais ce qu'il faudrait pas, c'est que après on reçoive quotidiennement dans notre boîte mail, des pubs... Parce que dès à ce moment là on va aller dans un endroit qui sera équipé comme ça, après on va avoir notre boîte mail remplie de publicités et de trucs comme ça » (couple, femme, 18-24 ans, employée ; homme, 18-24 ans, en recherche d'emploi).

D'autre part, la technologie employée est d'autant plus difficile à accepter que les applications associées par les interviewés sont chargées d'un imaginaire sécuritaire éloigné des fonctionnalités de médiation. À ces réticences s'ajoute l'effet sans doute non négligeable de la visite de l'exposition elle-même dont le thème visait notamment à sensibiliser les visiteurs aux techniques d'espionnage et de camouflage : "sur le principe, non, moi je trouve qu'il faut laisser ça à la justice, à l'armée... F : ... au service secret, ou des choses comme ça. Mais dans le courant, dans la vie civile, non laissez-nous vivre! (...) J'accepte pas du tout d'être tracée, j'habite à un endroit j'ai des relations, le reste, la technologie comme ça, j'aime pas ça moi » (couple, femme, 55-64 ans, prof. intermédiaire ; homme, +65 ans, retraité).

Au-delà de la technologie elle-même, plutôt méconnue et sur laquelle les visiteurs ont assez peu d'a priori dans le cadre du musée, ce sont les usages et applications existantes dans d'autres contextes qui suscitent de la méfiance (5). : "ça dépend de son utilisation, ce sont des technologies qui sont à double tranchant. (...) si c'est pour recevoir de la publicité ou faire vendre tel ou tel produit parce quion est passé à droite plutôt qu'à gauche... » (femme, 25-34 ans,cadre supérieur).

\section{Vie privée et intimité}

Le dispositif technique testé heurte l'identité des visiteurs dans le sens où des informations personnelles peuvent être capturées. Ce système apparaît donc comme un dispositif agressif ou tout du moins menaçant pour l'identité et l'intégrité des visiteurs qui se sentent «fliqués ». Certains revendiquent le droit de rester en dehors du système de médiation proposé, d'autres Ils souhaitent se tenir à l'écart ou encore se sentent même culpabilisés par la présence d'un système « inquisiteur » : " on est quand même des gens honnêtes ! [...] je suis pas bandit, je suis pas fiché, j'ai pas de casier judiciaire, non non laissez-nous tranquille! » (couple, femme, 55-64 ans, profession intermédiaire ; homme, +65 ans, retraité).

Face à cette pression d'un tel système, les interviewés revendiquent leur droit à l'intimité et à la vie privée : "on a tous, à un moment donné, notre petit jardin secret, et qu'on dévoile ce quion a envie de dévoiler, mais euh... Mais ouais à son insu.... » (femme, 2534 ans, éducatrice).

Au final, l'effet direct de l'exposition Ni vu ni connu se fait ressentir quand les visiteurs, interviewés à propos du dispositif intrusif de cette exposition, revendiquent leur droit au camouflage, au secret... bref leur droit à être ni vu ni connu : "c'est une technologie qui est complètement a contrario de ce qu'on a vu en fait, parce que là on cherche à prendre tous les renseignements sur ta personnalité ou même ton

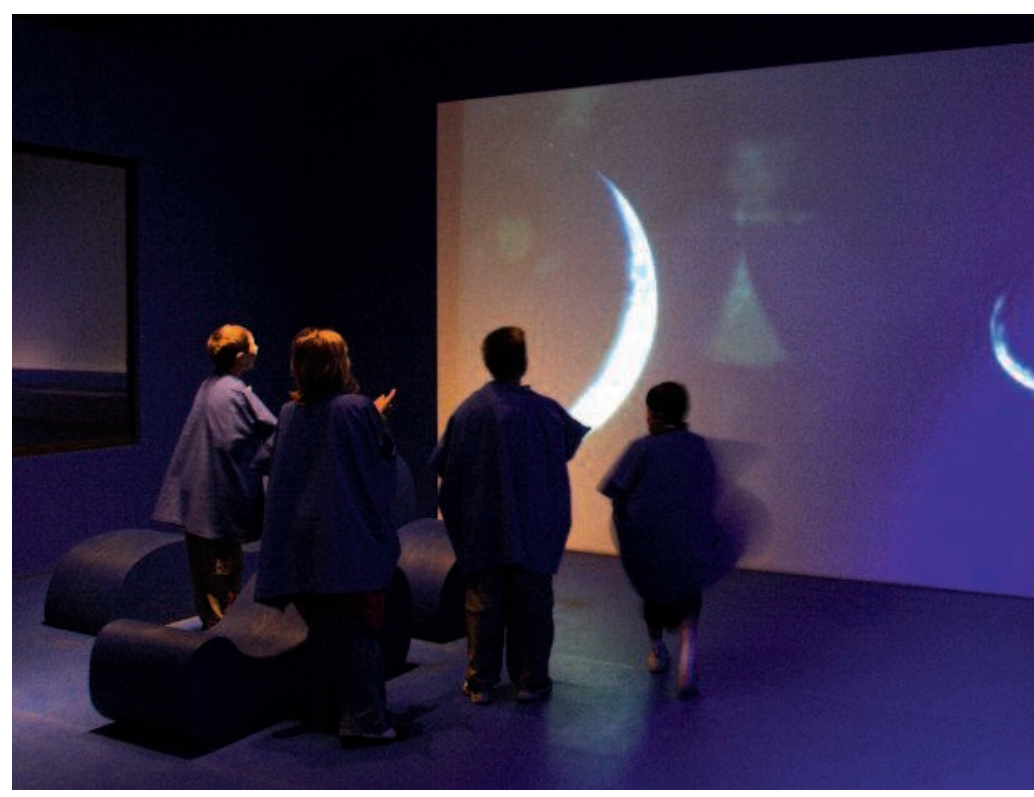

Des visiteurs, munis de leur cape font l'expérience de l'invisibilité. (c) Muséum-Département du Rhône/Jennifer Plantier 
physique, et on vient de passer une heure avec tout ce qui était Ni vu ni connu, tout ce que font les animanx, l'armée et tout pour se cacher, c'est complètement le contraire » (couple, femme, 55-64 ans, profession libérale ; homme, +65 ans, retraité).

\section{Pertinence du lien avec l'exposition}

Ainsi l'utilisation des technologies RFID doit se justifier par une véritable valeur ajoutée dans la médiation du contenu, avoir un lien clair avec la thématique et s'intégrer logiquement dans la scénographie. Pertinence et cohérence sont les règles d'utilisation des RFID pour préserver la relation de confiance avec le visiteur. On voit clairement les réticences ou formes d'opposition s'amenuiser lorsque les visiteurs établissent des liens avec le contenu thématique de l'exposition : " ... c'était une exposition "Ni vu ni connu", donc ça été un piège, en fait d'une certaine manière. Mais autrement en règle générale c'est une certaine violation de la personne " (couple, femme, 55-64 ans, prof. intermédiaire ; homme, +65 ans, retraité) ; " la carte ? ça m'a fait penser à... l'homme c'est un insecte. Il peut être contrôlé, il est dans une masse, il peut pas se distancier des autres » (couple : 18-24 ans ; étudiants).

Dans le cadre de l'exposition Ni vu ni connu, le scénario est accepté grâce au lien fort et évident avec la thématique. Ce ne serait pas forcément le cas avec d'autres thématiques ou scénographies.

D’autre part, le principe de la révélation de l'expérimentation à l'issue du parcours contribue à renforcer l'expérience subjective du musée. Ceci renvoie à lidée du visiteur acteur, plongé au cœur d'une expérience. Il s'agit d'une forme de médiation qui fait sens. En effet, le visiteur devient acteur au-delà d'une simple interaction dans le dispositif de médiation. "Prendre conscience » (expression employée par certains visiteurs ayant expérimenté le dispositif RFID de l'exposition) va au-delà de "savoir » ou d' "apprendre ». Ce type de médiation renforce le rôle du musée dans le transfert de la diffusion de connaissances : " moi je trowve ça intéressant parce qu'au contraire un musée a plutôt la fonction pédagogue, a priori, donc là c'est pour nous révéler, nous faire prendre conscience de certaines choses qui, dont on peut difficilement être conscient a priori si l'on ne maîtrise pas la technologie ou juste savoir. F2 : ça permet d'expliquer aux visiteurs : " regardez ce quion peut faire avec les technologies anjourd'hui ", si on s'en servait pas on pourrait absolument pas nous l'expliquer » (femmes, 25-34 ans /18-24 ans ; banque, étudiante droit).

Il s'agit d'un exemple typique d'amélioration qualitative dans le rapport au musée qui constitue une

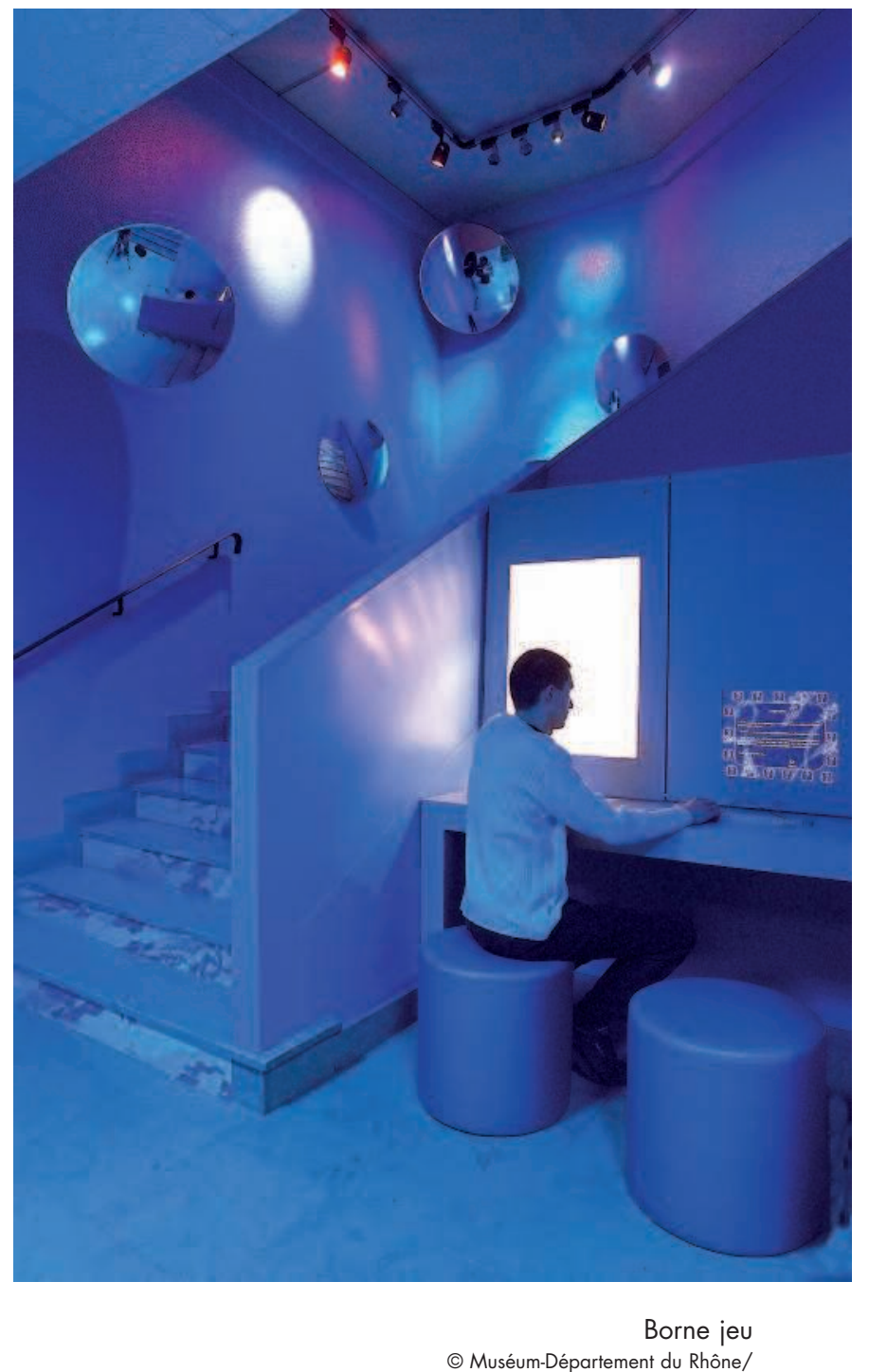

attente des visiteurs vis-à-vis de ce type de technologie. En utilisant ce type de procédé "immersif » dans ses outils de médiation, le musée va au-delà d'un transfert de savoirs en montrant les choses, il permet au visiteur d'expérimenter, de "voir par lui-même » pour acquérir, par l'expérience, de la connaissance plus que du savoir (au sens strictement pédagogique du terme). Par exemple, avec le dispositif expérimenté lors de l'exposition Ni vu ni connu le visiteur n'apprend pas seulement la notion de "flicage », il l'expérimente et connaît le sentiment d'être "fliqué » ou observé à son insu. Il se trouve ainsi sensibilisé à une application spécifique des RFID dont il perçoit le sens, la portée en contexte muséal ou étendu à d'autres domaines d'application. 


\section{La relation public/institution}

Pacte de confiance implicite avec le musée L'analyse des discours à partir de la verbalisation de l'expérience de visite fait apparaître clairement une différence «d'acceptabilité » pour la technologie RFID au musée par rapport à d'autres institutions bien identifiées par les visiteurs : commerces, industries culturelles, police. Les discours des visiteurs révèlent de façon récurrente un rapport de confiance « implicite » avec l'institution Musée. Dans la légitimité relative quils accordent à l'utilisation des rfid au musée, certains visiteurs vont jusqu’à établir une définition du musée au travers de ses missions : «F1 : Peut être parce que justement ils ont une vocation pédagogique, une vocation de recherche scientifique aussi quelque part, parce que c'est un établissement public alors bon ça dans notre pays à nous ça va, parce que c'est une démocratie. F2 : Oui y'a une certaine je dirais plus une certaine responsabilité dans un musée a priori. F1 : Voyez la télé par exemple. F1 : Comme c'est gratuit qu'on sait que c'est pas pour faire de l'argent que c'est pédagogique, c'est scientifique. F2 : ça cherche pas forcément à être au top de l'actualité, à attirer tout le monde, mais ça explore les sujets pas forcément évidents auxquels on n'aurait pas pensé. Y'a quand même une recherche un peu plus intellectuelle... » (femmes, 25-34 ans /18-24 ans ; banque, étudiante droit).

La bienveillance des visiteurs vis-à-vis du musée et leur tolérance du dispositif testé révèle un rapport complice avec l'institution et une disposition à coopérer avec le musée soumis à deux conditions principales : le besoin d'être informé des finalités de l'expérience pour se positionner vis-à-vis du développement de ce type d'application ; la connaissance des conditions dans lesquelles l'expérimentation pourrait se poursuivre.

\section{Prolongement de l'expérience}

Lidée de prolongement de l'expérience au-delà du parcours de visite a généré des points de vue ambivalents : d'une part, l'intérêt d'être informé à domicile perçu comme un service du musée permettant de garder une mémoire de la visite ; d'autre part, une crainte d'une communication ciblée avec des risques de dérive publicitaire. Le souhait de recevoir des informations actualisées, des liens web utiles liés à une expérience antérieure au musée, une thématique d'exposition paraît faible par rapport aux réticences exprimées par les interviewés : la crainte d'être assisté s'exprime dans les termes suivants «c'est pas le musée qui vient à nous c'est nous qui allons vers... »; « paresse intellectuelle », «ça infantilise »...
Les usagers sont peu favorables à cette idée d'une personnalisation de l'offre qui fige l'individu dans un profil défini. La spécificité du lieu culturel comme lieu de création tient justement à cette capacité de surprendre, de proposer des offres qui ne vont pas systématiquement dans le sens des attentes des publics, qui les surprennent, les amènent vers des territoires méconnus voire inconnus. La tentative de proposer des offres « sur mesure " s'inscrit en faux par rapport à la philosophie du projet culturel. Les visiteurs souscrivent à ce projet lorsqu'ils rejettent l'idée d'un musée qui utiliserait les technologies de personnalisation pour se contenter de leur offrir ce qu'ils demandent ou ce qui correspond à leur « profil ». La distinction s'opère plutôt entre ce qui relève des services et des moyens mis à disposition du visiteur et ce qui relève des produits (médiation, expositions...).

La proposition de poursuivre la relation musée/visiteur en dehors de l'institution, en dépassant les contraintes spatiales et temporelles de l'exposition (R. Topalian, 2006) dans le cadre d'une exploitation

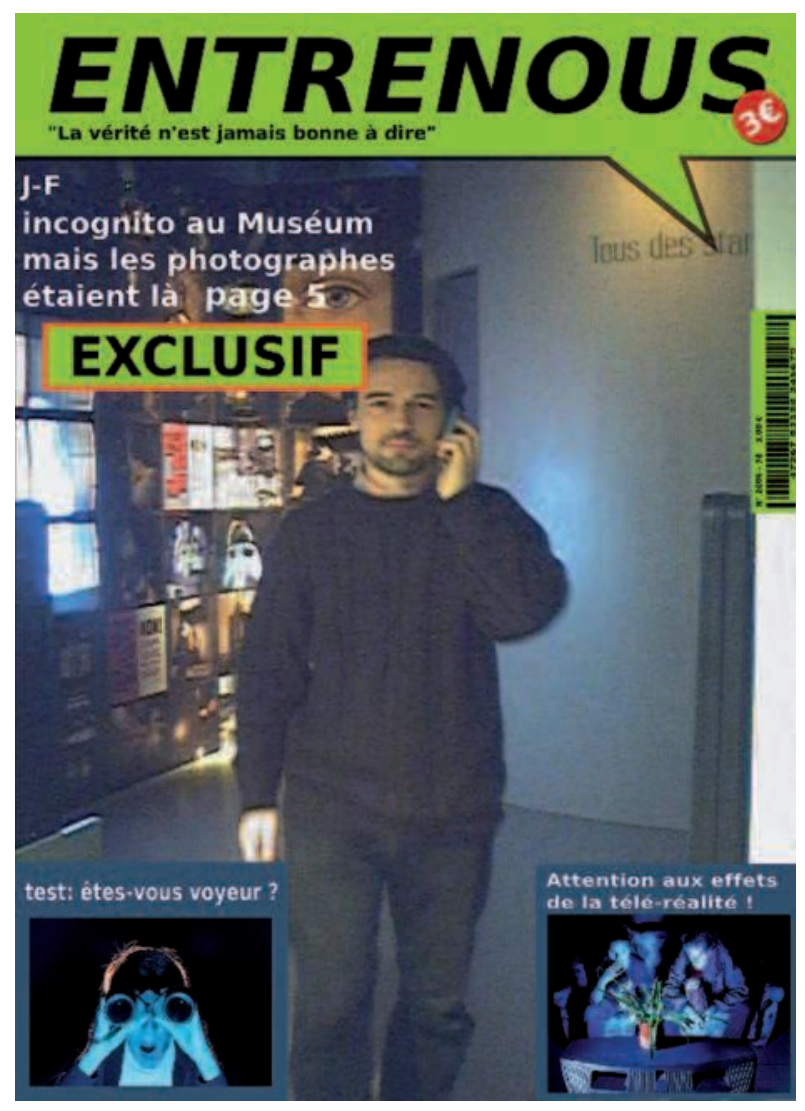

À l'issue de son parcours, le visiteur dépose sa carte dans la borne finale. Une fausse couverture de magazine " people» s'affiche alors, avec sa photographie prise à son insu dans «Le tunnel des paparazzi » et son pseudonyme renseigné lors de son passage à la borne interactive. (c) Muséum-Département du Rhône/J.-F. Salmon 
ultérieure de leur parcours de visite via un site Internet est jugée intéressante mais fait également émerger des craintes quant à l'utilisation des données personnelles (mél...) dont on ne maîtriserait pas le contrôle.

Le paradoxe tient au fait que le musée, s'il souhaite continuer à utiliser les nouvelles technologies d'information et communication de type « intelligence ambiante » devra s'appliquer à lui-même les leçons qu'il a souhaité donner aux visiteurs au cours de l'exposition $\mathrm{Ni}$ vu ni connu, c'est-à-dire un principe de précaution et de transparence. Si le pacte est respecté entre les visiteurs et le musée une relation ludique peut s'instaurer et les RFID peuvent faire partie de ce jeu si les règles sont claires. L'interactivité constitue l'une des exigences des visiteurs vis-à-vis du musée et les RFID bénéficient de cet a priori positif en tant que technologie de médiation. Certains visiteurs endossent spontanément une identité « joueuse » : "si je suis prévenue au début et que je joue le jeu, non pourquoi ? Je joue le jeu » (femme, 55-64 ans, retraitée ; avec son petit-fils).

\section{Conserver la mémoire de sa visite au musée ou permettre au musée d'interagir avec les usagers... quelles perspectives ?}

Comme nous le disent les visiteurs (" la technologie à sa place dans un musée. (...) on sera en retard sur son temps si y'en avait pas même »), le recours aux technologies de l'information contribue à inscrire l'institution muséale comme lieu d'expérimentation, ancré dans la vie actuelle en lui permettant d'être ou de rester " de son temps » et en offrant aux visiteurs une pratique contemporaine du musée.

Leurs points de vue rendent compte des caractéristiques de cette expérimentation jugée à la fois innovante, inéluctable (" on est obligé d'y arriver »...) et contribuant à renforcer la participation des publics grâce à de nouvelles formes d'interactivité.

Au-delà de l'interactivité qu'elle induit, les participants imaginent les atouts pour l'institution en termes de muséographie ou d'évaluation. Ils pensent que ces technologies peuvent améliorer la connaissance des publics, leur parcours, permettre d'identifier les zones d'attraction et de rétention ("voir qu'est-ce qui a plu... Qu'estce qui attire le plus de monde, là où les gens passent le plus vite ») afin d'adapter ensuite les propositions.

Au-delà de la technologie RFID, cette expérience permet de réfléchir plus largement au rôle des systèmes d'intelligence ambiante dans les musées. Bien que modeste au niveau des performances (RFID uniquement) le dispositif testé est représentatif de fonctionnalités typiques des systèmes d'intelligence ambiante, c'est-à-dire sensibles au contexte (le visiteur, les objets, l'environnement) et fonctionnant de manière « proactive et pervasive » : autonomie de fonctionnement, anticipation, transparent pour l'utilisateur... C'est donc la possibilité d'utiliser ce type de technologie « sensible au contexte » qui pose question. Il sera possible d'aller beaucoup plus loin dans l'interactivité et "l'immersion » dans les années à venir, grâce notamment à la possibilité d'utiliser des capteurs (mouvement, présence, ambiance et pourquoi pas état psychologique : plaisir/ennui... du visiteur $\left.{ }^{(6)}\right)$. Il faut envisager, avec ce type de technologies et de fonctionnalités associées, la possibilité de rendre le dispositif scénographique, voire les contenus eux-mêmes, sensibles et réactifs au contexte ou même à la situation sociale : identité et caractéristiques des visiteurs ou groupes de visiteurs.

Or le contexte dans le musée c'est aussi la thématique et la nature même de l'institution muséale. L'erreur évidente serait de " décontextualiser » l'utilisation de ce type de technologies par rapport au thème de l'exposition ou au lieu " musée ». Ce que montre notre expérience sur le dispositif $\mathrm{Ni} v u, n i$ сопnu c'est la pertinence du lien entre les technologies de médiation, les thématiques abordées et le rôle institutionnel du musée. L'équation à résoudre pour combiner ces facteurs est soumise à un paradoxe. On ne peut pas tout se permettre dans l'utilisation des nouvelles technologies au musée, parce que c'est le musée... En même temps, leur rôle est très prometteur et tout est possible (ou presque), parce que c'est le musée : un lieu qui permet le questionnement, l'anticipation, la distanciation, la réflexion (prise de conscience), le jeu...

\section{Notes}

(1) Portant sur les significations d'usage du dispositif RFID dans l'exposition Ni vu ni Connu. Paraître, disparaître, apparaître (Muséum, Lyon, 2006), l'étude a été conduite par la maison des Sciences de l'HommeAlpes et la cellule Évaluation du muséum de Lyon.

(2) Mallein, P. et Toussaint Y. (1994)

(3) L'enquête s'est déroulée la dernière semaine de présentation de l'exposition (semaine du 26 juin au 2 juillet 2006) auprès d'un échantillon aléatoire de visiteurs dont on a tenté de prendre en compte des critères de profils distincts en termes d'âge et d'accompagnement (seul, en famille, en couple, entre amis, avec enfants (18 visiteurs interrogés).

(4) Le spam, mot d'origine anglaise, désigne les communications électroniques massives, notamment de courrier électronique, sans sollicitation 